\title{
Commutative partial differential operators
}

\author{
Alex Kasman $^{\mathrm{a}, *}$, Emma Previato ${ }^{\mathrm{b}}$ \\ ${ }^{a}$ Department of Mathematics, College of Charleston, 66 George Street, Charleston, SC 29424-0001, USA \\ ${ }^{\mathrm{b}}$ Department of Mathematics, Boston University, 111 Cummington Street, Boston, MA 02215, USA
}

\begin{abstract}
In one variable, it is possible to describe explicitly the differential operators that commute with a given one, at least when the centralizer of the given operator has rank 1. So far, a generalization of the theory to several variables has been developed (inexplicitly) only for matrices, whose size increases with the number of variables. We propose to develop an algebraic theory of commuting partial differential operators (PDOs) by formulating a generalization of the one-variable techniques, in particular Darboux transformations and differential resultants. In this paper, we present a counter example to a one-variable feature of maximal-commutative rings and some facts, examples and questions on differential resultants. (C) 2001 Elsevier Science B.V. All rights reserved.
\end{abstract}

Keywords: Partial differential operators; Maximal-commutative rings; Differential resultant

\section{Introduction}

In the 1920s, when Burchnall and Chaundy [3,4] undertook their classification of commutative rings of differential operators there was no reason to expect an application in nonlinear dynamics. With hindsight, however, we see that they had discovered one important aspect of the modern theory of integrable systems: the spectral curve [20]. As we will explain in the next section, they even came quite close to discussing the commuting flows. Of course, it was not until the discovery of solitons and the integrability of the KdV equation that this theory would be rediscovered and placed in an appropriate context. In particular, the Burchnall-Chaundy correspondence between ordinary differential operators (ODOs) and algebraic curves provides an algebraic approach to handling the inverse spectral problem for the finite-gap operators, with the spectral data being encoded in the spectral curve and an associated line bundle. Moreover, just as a classical integrable system has an action-angle map which linearizes its flow, the (quasi-)periodic solutions to the soliton equations are linearized by the Buchnall-Chaundy correspondence in the sense that the spectral curve is invariant and the line-bundle changes according to a linear flow on its Jacobian variety [14].

In this paper, we will discuss some aspects of the generalization of the Burchnall-Chaundy problem to the higher dimensional case of a commutative ring of partial differential operators (PDOs). Though this difficult problem is far from completely solved, it is clearly of interest not only for its own sake but for its potential connections to integrable systems. In fact, the study of commutative rings of PDOs and their algebro-geometric classification is already of

\footnotetext{
* Corresponding author.

E-mail addresses: kasman@math.cofc.edu (A. Kasman), ep@math.bu.edu (E. Previato).
} 
interest in the context of quantum integrable systems where they are literally the commuting Hamiltonians of a finite dimensional system $[2,6,12]$. In addition, there have been several attempts at generalizations of the KP hierarchy of integrable PDEs to the case of a system written as a Lax pair in terms of PDOs [17,18,21] and one would expect commutative rings of PDOs to play an analogous role in that situation.

In one variable, there exists a satisfactory classification of rings of differential operators that are (maximal) commutative. In several variables, even the simplest generalizations seem to be unknown and in this report we give examples and pose questions that may suggest a theory to be developed. To motivate what we do, we briefly recall the one-variable case and selected results in several variables.

We will focus our attention here on two particular aspects of the generalization of Burchnall-Chaundy theory to the higher dimensional case. Specifically, we address the question of whether one may expect a maximal-commutative ring of PDOs to be the coordinate ring of an affine algebraic variety (as in the one-dimensional case where it is always the coordinate ring of an affine curve) and whether the differential analog of the resultant provides a means for determining the polynomial equations satisfied by an algebraically dependent set of commuting PDOs.

\subsection{The one-dimensional case}

In the one-variable case with analytic coefficients the classification was found by Burchnall and Chaundy $[3,4]$ in the 1920s by essentially formal methods of differential algebra and some algebraic geometry. They stated the problem quite simply: characterize the operators $L=\partial^{n}+u_{n-2}(x) \partial^{n-2}+\cdots+u_{0}(x)$ whose centralizer is not isomorphic to the polynomials in one variable. Indeed, for $L$ general enough, the only differential operators that commute with it will be the polynomials in $L$ with constant coefficients. Here, we consider the centralizer of $L$ in the ring of ODOs, where multiplication is composition and therefore obeys the defining rule: $[\partial, x]=1$; with a view to introducing several variables below, we write $\partial=\partial / \partial x$ although here we are studying the one-variable case. The question is an algebraic one, and we will not specify the function space where the coefficients $u_{i}(x)$ live. Noting that the centralizer of an ODO is itself commutative, Burchnall and Chaundy proceeded to show that it is a ring of 'dimension' 1 , in other words, the affine ring of an algebraic curve (this observation goes back at least to Schur but Burchnall and Chaundy appear to reproduce it independently). The curve can be seen concretely if we consider the ring $\mathbb{C}[L, B]$ generated by a commuting pair, where $B=\partial^{m}+v_{m-2}(x) \partial^{m-2}+\cdots+v_{0}(x)$. The curve is then the set of points $(\lambda, \mu)$, such that $(L-\lambda, B-\mu)$ have a common solution $f(x)$, or equivalently such that the determinant of the $(n+m) \times(n+m)$ "differential resultant" matrix [19], a non-zero polynomial $p \in \mathbb{C}[\lambda, \mu]$, is equal to zero. The commuting operators satisfy (identically in $x$ ) the algebraic equation $p(L, B)=0$. Moreover, in the "rank-1 case" $\operatorname{gcd}(n, m)=1$ [20], one has that $p(\lambda, \mu)=0$ is the affine equation of the spectral curve. More generally, if $A$ is any commutative ring of ODOs whose rank is 1 (i.e., $A$ contains two elements of coprime orders), then $A$ is isomorphic to a finitely generated algebra $\mathbb{C}\left[x_{1}, x_{2}, \ldots, x_{s}\right] / I$, where the ideal $I$ contains at least $(s-1)$ independent equations (in algebro-geometric terminology, $\operatorname{Spec} \mathcal{A}$ is an affine curve).

Burchnall and Chaundy's contribution is deeper in fact, because they were able to classify all the (rank-1) isospectral algebras, namely those associated to isomorphic isospectral curves. They started with the observation that the (Darboux) transformation $A \mapsto G A G^{-1}$ (where $G=\partial-\left(f\left(x, P_{0}\right) / f^{\prime}\left(x, P_{0}\right)\right)$ is the greatest common divisor of the operators in $A$, for a common eigenfunction $f$ which depends on a given point $P_{0}$ of the curve, explicitly in the above example $L f(x)=\lambda f(x), B f(x)=\mu f(x), f\left(x_{0}\right)=1$, a normalization) does not change the curve but changes the set of points $P_{1}, \ldots, P_{g}$, where the common eigenfunction $f(x, P)$ of the ring has a pole. In classical geometric language, the divisor $P_{1}+\cdots+P_{g}$, changes into $P_{1}+\cdots+P_{g}+\infty-P_{0}$ (the affine curve is completed by adding a smooth point $\infty$ ) and the set of divisors up to linear equivalence is called the Jacobi variety of the curve. This was then Burchnall and Chaundy's crowning result: the set of isospectral algebras associated to a given spectral curve $X$ of genus $g$ is an open subset of a complex torus of dimension $g$, namely the Jacobi variety 
of the curve; moreover, translation in $x$ is a linear vector field on the torus, which they determined explicitly in abelian coordinates. In the 1970s, and in consequence of the renewed interest in the spectral problem promoted by the Zakharov-Shabat scattering problem, Krichever [14] solved the inverse spectral problem explicitly (using the Riemann theta function), associating to a curve and a divisor (up to choice of certain normalizations) a commutative algebra $A$ of ODOs.

\subsection{Higher dimensional case}

In view of the success in the one-dimensional situation and the potential applications in the higher dimensional case, it is natural to ask at least the following questions in several variables:

1. Is $\operatorname{Spec} \mathcal{A}$ an affine variety of dimension $N$ for any maximal-commutative ring $\mathcal{A}$ of PDOs in $N$ variables?

Without entering the technical definitions of algebraic geometry, a major implication of this would be for $A$ to be finitely generated as a $\mathbb{C}$ algebra, which we disprove below.

2. For a ring $\mathcal{A}$ with generators $L_{i}(1 \leq i \leq N+1)$, what is the relationship between the differential resultant of $L_{i}-\lambda_{i}$ and the equations of such a variety?

More ambitiously, of course, one would ask for isospectral flows, a complete classification, and the explicit inverse spectral problem, in increasing order of difficulty. A beautiful generalization of the Burchnall-Chaundy theory was given by Nakayashiki $[17,18]$ using the Fourier-Mukai transform. He associates commutative rings in $N$ variables to a suitable $N$-dimensional abelian variety and some additional choices, one ring for each element of the Picard variety of the abelian variety. But these are not scalar operators, rather they have $(N ! \times N !)$ matrix coefficients.

In this report, we give a negative answer to question (1) by using techniques developed in [13] and we offer some observations, natural conjectures, and a strategy to treat (2). We deal with the scalar case only.

\section{Maximal-commutative rings}

It is well known that the commutative rings of ODOs are (finitely generated) coordinate rings for algebraic curves. This forms the foundation of the Burchnall-Chaundy theory of such rings $[3,4,20]$. In contrast, very little is known about the algebro-geometric structure of commutative rings of PDOs. A question of interest is to address the problem of whether every commutative subring is contained in a (larger) commutative ring requiring only a finite number of generators over $\mathbb{C}$. Such a result is relevant, for instance, to the algebro-geometric investigations of quantum integrable systems $[2,12]$.

In this section, we use techniques from soliton theory (namely Darboux transformation and Baker-Akhiezer functions) to study the structure of certain commutative rings of differential operators. We are able to show that these rings are maximal in the sense that they are not contained in any larger commutative subrings of the ring of differential operators. Then, in one example, we study the structure of this ring more closely and note that it cannot be generated by a finite number of elements as a $\mathbb{C}$ algebra and hence is not the coordinate ring of an affine algebraic variety.

Notation. Let $\mathcal{D}=\mathbb{C}\left(x_{1}, \ldots, x_{n}\right)\left[\partial_{1}, \ldots, \partial_{n}\right]$ be the ring of rational coefficient differential operators in $n$ variables, where $\partial_{n}=\partial / \partial x_{n}$ is differentiation and composition is determined by action on functions. It will be useful to refer also to $\mathcal{D}_{0}=\mathbb{C}\left[\partial_{1}, \ldots, \partial_{n}\right] \subset \mathcal{D}$ (the constant coefficient differential operators) as well as $\Psi \mathcal{D}$, a ring of pseudodifferential operators ([22] or [11] for notation but here regarded as formal objects) containing $\mathcal{D}$ as well as the inverse of the particular operator $K \in \mathcal{D}$ which will be important below and $\Psi \mathcal{D}_{0}$, the constant coefficient pseudodifferential operators $\mathbb{C}\left(\left(\partial_{1}^{-1}, \ldots, \partial_{n}^{-1}\right)\right)$. 


\subsection{What commutes with many constant coefficient operators?}

In general, it is difficult to address the question of whether a commutative ring of PDOs is maximal. The key observation that allows us to do it here is the following: although the centralizer of a single constant coefficient operator $p\left(\partial_{1}, \ldots, \partial_{n}\right)$ will contain non-constant coefficient operators, an operator commutes with all constant coefficient multiples of $p\left(\partial_{1}, \ldots, \partial_{n}\right)$ if and only if it also has constant coefficients.

Lemma 2.1. Let $p \in \mathcal{D}_{0}$ be a non-zero constant coefficient differential operator. Then any operator $L \in \Psi \mathcal{D}$ which commutes with $p$ as well as all operators $P_{i}:=\partial_{i} \circ p\left(\partial_{1}, \ldots, \partial_{n}\right)(1 \leq i \leq n)$ is also constant coefficient $(i . e$. , $\left.L \in \Psi \mathcal{D}_{0}\right)$.

Proof. Let us suppose that $[p, L]=\left[P_{i}, L\right]=0$. Then, since $p \circ L=L \circ p$ and $p \circ \partial_{i}=\partial_{i} \circ p$ it follows that:

$$
0=\left[P_{i}, L\right]=\partial_{i} \circ p \circ L-L \circ \partial_{i} \circ p=\partial_{i} \circ L \circ p-L \circ p \circ \partial_{i}=\left[L \circ p, \partial_{i}\right] .
$$

Then, we note that letting $L \circ p=\sum f_{\alpha}\left(x_{1}, \ldots, x_{n}\right) \partial_{1}^{\alpha_{1}} \cdots \partial_{n}^{\alpha_{n}}$ be a series representation for this pseudodifferential operator, then

$$
\left[\partial_{i}, L \circ p\right]=\sum f_{\alpha}^{\prime}\left(x_{1}, \ldots, x_{n}\right) \partial_{1}^{\alpha_{1}} \cdots \partial_{n}^{\alpha_{n}}
$$

where prime denotes differentiation with respect to $x_{i}$. Hence, if $L \circ p$ commutes with each $\partial_{i}$, then $L \circ p$ has constant coefficients. However, this provides a linear relation between any coefficient of $L$ and certain higher ones. This is only possible if the coefficients are all constant; indeed if they were not, one can get a contradiction easily, first by assigning an (e.g., lexicographic) order to the monomials, then multiplying the two monomials in $L, p$ that contain the highest power of a given variable whose coefficient in $L$ is non-constant; the product cannot be cancelled.

\subsection{A maximal-commutative ring from Darboux transformation}

Here, we will consider a special class of commutative rings of differential operators for which we are able to demonstrate maximality using the results of the previous subsection. Suppose that the constant coefficient operator $p \in \mathcal{D}_{0}$ factors as

$$
p\left(\partial_{1}, \ldots, \partial_{n}\right)=L \circ K, \quad L, K \in \mathcal{D} .
$$

(See [1,13] for a discussion of some methods for achieving such factorizations.) Then the method of Darboux transformation commonly used in the study of integrable systems [8,16] is to consider the (more complicated) operator

$$
P:=K \circ L=K \circ p \circ K^{-1}
$$

which shares many features with $p$ since the two operators are conjugate. For instance, one may try to conjugate other constant coefficient operators by $K$ to produce operators that commute with $P$. In fact, given any constant coefficient operator $r\left(\partial_{1}, \ldots, \partial_{n}\right) \in \mathcal{D}_{0}$ it follows that $\left[K \circ r \circ K^{-1}, P\right]=0$. However, although $K \circ r \circ K{ }^{-1} \in \Psi \mathcal{D}$ there is no reason to expect that it is in $\mathcal{D}$. The content of the Theorem 2.1 is the statement that the ring of all differential operators commuting with $P$ which are produced in this way is a maximal-commutative ring.

Notation. Let $K \in \mathcal{D}$ be a differential operator and define

$$
R_{0}(K):=\left\{r \in \mathcal{D}_{0} \mid K \circ r\left(\partial_{1}, \ldots, \partial_{n}\right) \in \mathcal{D} \circ K\right\} \subset \mathcal{D}_{0}
$$


to be the subring of elements $r \in \mathcal{D}_{0}$ such that $K \circ r$ has $K$ as a right factor. Then, the ring

$$
R(K):=\left(K \circ \mathcal{D}_{0} \circ K^{-1}\right) \cap \mathcal{D}=K \circ R_{0}(K) \circ K^{-1}
$$

is a commutative subring of $\mathcal{D}$. In general, it will be the case that $R(K)=\mathbb{C}$ is trivial, but if $K$ is chosen to be a non-constant operator satisfying (1) then $R(K)$ will contain differential operators.

Theorem 2.1. Let $K \in \mathcal{D}$ be a differential operator which is the right factor of some constant coefficient operator $p=L \circ K \in \mathcal{D}_{0}$. Then if $R^{\prime}$ is a commutative ring such that

$$
R(K) \subset R^{\prime} \subset \mathcal{D},
$$

it follows that $R(K)=R^{\prime}$. In other words, $R(K)$ is a maximal-commutative subring of $\mathcal{D}$.

Proof. Let us suppose that $Q \in \mathcal{D}$ commutes with every element of $R(K)$. We must show that $Q$ is already in $R(K)$. Note that since $p=L \circ K$, one automatically has that $P_{i}:=\partial_{i} \circ p$ is in $R_{0}(K)$. Thus, we know that

$$
\left[K \circ P_{i} \circ K^{-1}, Q\right]=0 \text { for } 1 \leq i \leq n,
$$

and hence conjugation in the ring of pseudodifferential operators gives

$$
\left[P_{i}, K^{-1} \circ Q \circ K\right]=0, \quad 1 \leq i \leq n .
$$

By Lemma 2.1, this implies that

$$
K^{-1} \circ Q \circ K \in \Psi \mathcal{D}_{0},
$$

and so it is clear that $Q$ is of the form $K \circ q\left(\partial_{1}, \ldots, \partial_{n}\right) \circ K^{-1}$ for some constant coefficient pseudodifferential operator $q \in \Psi \mathcal{D}_{0}$.

However, we can also see that $q \in \mathcal{D}_{0} \subset \Psi \mathcal{D}_{0}$ is a constant coefficient differential operator. To show this we introduce a normalized common eigenfunction

$$
\psi\left(x_{1}, \ldots, x_{n}, z_{1}, \ldots, z_{n}\right)=\frac{1}{g\left(z_{1}, \ldots, z_{n}\right)} K\left[\mathrm{e}^{x_{1} z_{1}+\cdots+x_{n} z_{n}}\right],
$$

where the polynomial $g$ is to be defined below. Note that regardless of the choice of $g$, we have by construction that

$$
K \circ L[\psi]=p\left(z_{1}, \ldots, z_{n}\right) \psi, \quad Q[\psi]=q\left(z_{1}, \ldots, z_{n}\right) \psi .
$$

One can write the function $K\left[\exp \left(\sum x_{i} z_{i}\right)\right]$ in the form

$$
K\left[\mathrm{e}^{x_{1} z_{1}+\cdots+x_{n} z_{n}}\right]=\left(\frac{\sum_{\alpha=1}^{N} \rho_{\alpha}\left(z_{1}, \ldots, z_{n}\right) \sigma_{\alpha}\left(x_{1}, \ldots, x_{n}\right)}{\sigma_{0}\left(x_{1}, \ldots, x_{n}\right)}\right) \mathrm{e}^{x_{1} z_{1}+\cdots+x_{n} z_{n}},
$$

where $\rho_{\alpha}$ are all non-zero polynomials in $z_{1}, \ldots, z_{n}$ and $\sigma_{\alpha}$ are distinct, non-zero monomials in $x_{1}, \ldots, x_{n}$. We choose $g\left(z_{1}, \ldots, z_{n}\right)$ to be the highest common factor of the polynomials $\rho_{\alpha}\left(z_{1}, \ldots, z_{n}\right)$. We have thus constructed $\psi$, so the product $f\left(z_{1}, \ldots, z_{n}\right) \psi$ is holomorphic in each $z_{i}$ for $f \in \mathbb{C}\left(z_{1}, \ldots, z_{n}\right)$ if and only if $f \in \mathbb{C}\left[z_{1}, \ldots, z_{n}\right]$ is actually a polynomial.

Then notice that for any $M \in \mathcal{D}$ one still has that $M[\psi]$ is holomorphic in $z_{i}$. In particular, $M[\psi]$ is always a polynomial in $\mathbb{C}\left(x_{1}, \ldots, x_{n}\right)\left[z_{1}, \ldots, z_{n}\right]$ multiplied by the exponential function $\exp \sum x_{i} z_{i}$. Putting this all together, since we have already seen that $Q[\psi]=q\left(z_{1}, \ldots, z_{n}\right) \psi$, we conclude that $q\left(\partial_{1}, \ldots, \partial_{n}\right) \in \mathcal{D}_{0}$ is a constant coefficient differential operator. 
Finally, if $q \in \mathcal{D}_{0}$ has the property that $K \circ q \circ K^{-1} \in \mathcal{D}$, then this implies that $q \in R_{0}(K)$ and hence that $Q \in R(K)$ in the first place. So no operators outside of $R(K)$ have the property that they commute with every element of $R(K)$.

\subsection{An explicit example}

It is still not clear from Theorem 2.1 whether the maximal-commutative subrings of $\mathcal{D}$ constructed by Darboux transformation require an infinite number of generators over $\mathbb{C}$. Certainly in trivial cases (e.g., $K \in \mathcal{D}_{0}$ ), the resulting ring may require only a finite number of generators. But it would be nice to prove that this is always the case or alternatively to observe at least one example which does not. By considering a particular example in detail here, we achieve the latter.

\subsubsection{A subring of $\mathbb{C}[x, y]$}

Notation. Let $\mathcal{R}_{\lambda} \subset \mathbb{C}[x, y](0 \neq \lambda \in \mathbb{C})$ be the subset

$$
\mathcal{R}_{\lambda}=\left\{q(x, y) \in \mathbb{C}[x, y] \mid q_{x}\left(z, \frac{\lambda}{z}\right)=q_{y}\left(z, \frac{\lambda}{z}\right)=q_{x y}\left(z, \frac{\lambda}{z}\right) \equiv 0\right\} .
$$

In other words, $\mathcal{R}_{\lambda}$ is the set of polynomials $q \in \mathbb{C}[x, y]$, such that $q_{x}, q_{y}$ and $q_{x y}$ all have a factor of $x y-\lambda$. Note that $\mathbb{C} \subset \mathcal{R}_{\lambda}$ and more importantly that if $q_{1}, q_{2} \in \mathcal{R}_{\lambda}$ are two such polynomials, then $q_{1}+q_{2} \in \mathcal{R}_{\lambda}$ and $q_{1} q_{2} \in \mathcal{R}_{\lambda}$. This obviously gives us the following lemma.

Lemma 2.2. $\mathcal{R}_{\lambda}$ is a proper subring of $\mathbb{C}[x, y]$ containing $\mathbb{C}$ as well as every polynomial of the form $\rho(x, y)(x y-\lambda)^{3}$ for $\rho \in \mathbb{C}[x, y]$.

It will be shown below that for a particular choice of $K \in \mathcal{D}$ the maximal-commutative subring $R(K) \subset \mathcal{D}$ is isomorphic to $\mathcal{R}_{\lambda}$. Therefore, it is interesting to note that this ring requires an infinite number of generators over $\mathbb{C}$.

Lemma 2.3. The ring $\mathcal{R}_{\lambda}$ has the form $\mathbb{C}\left[\omega_{1}(x y-\lambda)^{3}, \omega_{2}(x y-\lambda)^{3}, \ldots\right]$, where $\left\{\omega_{i}\right\}$ is any basis of $\mathbb{C}[x, y]$ as a vector space. In particular, $\mathcal{R}_{\lambda}$ is not finitely generated.

Proof. We must show that a polynomial $q \in \mathbb{C}[x, y]$ is in $\mathcal{R}_{\lambda}$ if and only if it is of the form $g(x, y)(x y-\lambda)^{3}+c$ for some $g \in \mathbb{C}[x, y]$ and $c \in \mathbb{C}$. Clearly, such a $q$ is an element of $\mathcal{R}_{\lambda}$. Conversely, let us suppose that $q \in \mathcal{R}_{\lambda}$ and therefore $q_{x}=(x y-\lambda) r(x, y)$. Then, since

$$
q_{x y}=x r(x, y)+(x y-\lambda) r_{y}(x, y)
$$

also has a factor of $x y-\lambda$, one finds that $r$ has a factor of $x y-\lambda$ and hence $q_{x}$ actually has a factor of $(x y-\lambda)^{2}$. (Similarly, for $q_{y}$.)

Now we have that $q_{x}=(x y-\lambda)^{2} g(x, y)$ for some $g \in \mathbb{C}[x, y]$. Then, integrating by parts with respect to $x$, one has

$$
q(x, y)=\frac{1}{3 y}\left[(x y-\lambda)^{3} g(x, y)-\int(x y-\lambda)^{3} g_{x}(x, y) \mathrm{d} x\right] .
$$

Continuing to integrate by parts (choosing always to integrate $(x y-\lambda)^{j}$, so that one gets higher powers of $x y-\lambda$ and higher derivatives of $g$ ) one gets a finite sum (since a high enough derivative of $g$ will eventually vanish) of terms each having a factor of $(x y-\lambda)^{3}$, plus a constant of integration at the end. 
Now we note that $\mathcal{R}_{\lambda}$ cannot be constructed by a finite number of generators over $\mathbb{C}$. We provide an argument by contradiction; we are grateful to a referee for suggesting a more thorough argument than our original one. Let $t=x y-\lambda$, treat $x, t$ as independent variables, and argue in $\mathbb{C}[x, 1 / x, t]$. Suppose w.l.o.g. that $w_{i j}=x^{i} y^{j} t^{3}=$ $x^{i}(\lambda / x+t / x)^{j} t^{3}=\sum_{p=0}^{j} c_{j p} x^{i-j} t^{p+3}$, with $i, j<N$, are generators. Then for $x^{k} t^{3}$ to be expressed as a product of $x^{i-j} t^{p+3}$, the product must have only one term: $p=0$ and $k=i-j \leq i<N$, which is a contradiction for $k>N$.

\subsubsection{Isomorphism to a ring of differential operators}

Let us use the notation of the preceding subsection to describe a maximal-commutative ring of differential operators. For this example, we will be working in two dimensions only, so $n=2$. The constant coefficient differential operator which we will factor is $p\left(\partial_{1}, \partial_{2}\right)=\left(\partial_{1} \partial_{2}-\lambda\right)^{3}(\lambda \in \mathbb{C})$ which factors as $p=L \circ K$ with

$$
K=x_{1} x_{2}\left(\partial_{1} \partial_{2}-\lambda\right) \circ \frac{1}{x_{1} x_{2}},
$$

and

$$
\begin{aligned}
L= & \partial_{1}^{2} \partial_{2}^{2}+\frac{1}{x_{1}} \partial_{1} \partial_{2}^{2}-x_{1}^{-2} \partial_{2}^{2}+\frac{1}{x_{2}} \partial_{1}^{2} \partial_{2}+\frac{1-2 \lambda x_{1} x_{2}}{x_{1} x_{2}} \partial_{1} \partial_{2} \\
& +\frac{-1-\lambda x_{1} x_{2}}{x_{1}^{2} x_{2}} \partial_{2}-x_{2}^{-2} \partial_{1}^{2}+\frac{-1-\lambda x_{1} x_{2}}{x_{1} x_{2}^{2}} \partial_{1}+\lambda^{2}+\frac{1}{x_{1}^{2} x_{2}^{2}}+\frac{\lambda}{x_{1} x_{2}} .
\end{aligned}
$$

Lemma 2.4. A constant coefficient operator $q\left(\partial_{1}, \ldots, \partial_{n}\right) \in \mathcal{D}_{0}$ is an element of $R_{0}(K)$ if and only if the function

$$
\psi\left(x_{1}, x_{2}, z\right):=x_{1} x_{2} \mathrm{e}^{x_{1} z+x_{2}(\lambda / z)}
$$

is in the kernel of the operator $K \circ q$ for all values of $z \in \mathbb{C}$.

Proof. One direction is especially simple. If $K \circ q=Q \circ K$, then

$$
K \circ q\left[x_{1} x_{2} \mathrm{e}^{x_{1} z+x_{2}(\lambda / z)}\right]=Q \circ x_{1} x_{2}\left(\partial_{1} \partial_{2}-\lambda\right)\left[\mathrm{e}^{x_{1} z+x_{2}(\lambda / z)}\right] \equiv 0 .
$$

Conversely, let us suppose that $K \circ q$ annihilates this function. This means that $M:=K \circ q \circ x_{1} x_{2}$ applied to $\exp \left(x_{1} z_{1}+x_{2} z_{2}\right)$ is zero for all $z_{1} z_{2}-\lambda=0$. But, note that $M$ applied to this exponential results in a polynomial in $z_{i}$ with coefficients in $\mathbb{C}\left(x_{1}, x_{2}\right)$ multiplied by an exponential. This product vanishes on $z_{1} z_{2}-\lambda=0$ if and only if the polynomial has a factor of $z_{1} z_{2}-\lambda$ which implies that

$$
M=Q \circ x_{1} x_{2}\left(\partial_{1} \partial_{2}-\lambda\right)
$$

for some $Q \in \mathcal{D}$. Multiplying this equation by $1 / x_{1} x_{2}$ on the right proves the lemma.

Using this lemma and the previous theorem, as well as the bispectrality $[9,10]$ of the constant coefficient operators, we demonstrate an isomorphism between $R(K)$ and $\mathcal{R}_{\lambda}$.

Theorem 2.2. The ring $R(K)$, known to be maximal commutative by the preceding theorem, is isomorphic to the ring $\mathcal{R}_{\lambda}$ (cf. Lemma 2.2).

Proof. Using Lemma 2.4 and Theorem 2.1, we know that $R(K)$ is isomorphic to the ring

$$
R_{0}(K)=\left\{q \in \mathcal{D}_{0} \mid K \circ q\left[x_{1} x_{2} \mathrm{e}^{x_{1} z+x_{2}(\lambda / z)}\right] \equiv 0\right\} .
$$


However, since $\partial_{z_{i}}:=\left(\partial / \partial z_{i}\right)$ commutes with differential operators in the variables $x_{i}$, this property is equivalent to saying that

$$
\partial_{z_{1}} \partial_{z_{2}}\left[K \circ q\left[\mathrm{e}^{x_{1} z_{1}+x_{2} z_{2}}\right]\right] \equiv 0 \quad \forall z_{1} z_{2}-\lambda=0 .
$$

This can be written as differential equations for $q$ by applying all of these differential operators, clearing the denominator by multiplying by a polynomial in $x_{1}, x_{2}$ and looking at the coefficients of each monomial in $x_{i}$. These will be differential expressions for polynomials in $z_{1}$ and $z_{2}$ including $q$ which must vanish on $z_{1} z_{2}-\lambda$. For this to happen, it is necessary and sufficient that $q_{x}, q_{y}$ and $q_{x y}$ all have $z_{1} z_{2}-\lambda$ as a factor.

\section{Resultants of commuting differential operators}

Any set of $n+1$ commuting PDOs in $n$ variables must satisfy a non-trivial algebraic equation (cf., e.g., the Lemma of Section 1.1 in [2]). Is it possible to use linear algebra to determine this relationship (as is generally done in the case $n=1$ )? In this section, we give a definition of resultants for PDOs (cf. [5]) including "spectral parameters" and indicate their role in this regard.

\subsection{Definitions}

Fix $0<n \in \mathbb{N}$ and denote by $\Omega^{d}$ the $\left(\begin{array}{c}n+d \\ n\end{array}\right)$-component vector

$$
\Omega^{d}=\left(\omega_{1}^{d}, \omega_{2}^{d}, \ldots\right)
$$

where $\omega_{i}^{d}$ run over all monomial, monic differential operators in the variables $x_{1}, \ldots, x_{n}$ of degree less than or equal to $d$. In other words

$$
\omega_{i}^{d} \in\left\{\partial_{1}^{\alpha_{1}} \ldots \partial_{n}^{\alpha_{n}}: \alpha_{i} \in \mathbb{N}, \sum \alpha_{i} \leq d\right\} .
$$

By writing it as a vector, we are supposing that they have an ordering. Such an ordering is a choice, not determined canonically, but the particular choice is not important to the following. Then, for any differential operator $L$ of order $d$ or less, we denote by $\overrightarrow{\mathbf{v}}_{d}(L)$ the vector whose $i$ th entry is the coefficient of $\omega_{i}^{d}$ in $L$. In particular, $L=\overrightarrow{\mathbf{v}}_{d}(L) \cdot \Omega^{d}$. Let $L_{1}, \ldots, L_{n+1}$ be differential operators in the variables $x_{1}, \ldots, x_{n}$ having orders $l_{1}, \ldots, l_{n+1}$, respectively.

Let $N:=-n+\sum l_{i}$ and construct the matrix $R_{\mu}=R_{\mu}\left(L_{1}, \ldots, L_{n+1}\right)$ whose rows are $\overrightarrow{\mathbf{v}}_{N}\left(\omega_{j}^{N-l_{i}} \circ\left(L_{i}-\mu_{i}\right)\right)$ for all $1 \leq i \leq n+1$ and all $1 \leq j \leq\left(\begin{array}{c}n+\underset{n}{N}-l_{i} \\ n\end{array}\right)$. We call any maximal minor determinant of $R_{\mu}$ a partial $\mu$ -shifted differential resultant. Note that each partial $\mu$-shifted differential resultant is a polynomial in the variables $\mu_{i}(1 \leq i \leq n+1)$ with coefficients that may depend on $x_{j}(1 \leq j \leq n)$.

Note. In the case $L_{i} \in \mathbb{C}\left[\partial_{1}, \ldots, \partial_{n}\right]$, taking the greatest common divisor of all of these maximal minor determinants is equivalent to calculating the polynomial resultant [15]; while for ODOs with variable coefficients the definition reproduces the differential resultant used to construct the spectral curve $[19,20]$. The definition of the differential resultant of the operators $L_{i}$ given in [5] is, in our terminology, a particular partial $\mu$-shifted differential resultant of the operators $L_{i}$ with all $\mu_{i}=0$.

As in the polynomial case, an important observation is the fact that the partial resultants can be achieved as linear combinations of the original operators. 
Lemma 3.1. Any partial $\mu$-shifted differential resultant of the operators $L_{1}, \ldots, L_{n+1}$ can be written as

$$
\sum_{i=1}^{n+1} D_{i} \circ\left(L_{i}-\mu_{i}\right)
$$

for some PDOs $D_{i}$ with coefficients depending on $\mu_{j}(1 \leq j \leq n+1)$ and $x_{k}(1 \leq k \leq n)$.

Proof. Let $j$ be the integer $1 \leq j \leq\left(\begin{array}{c}n+N \\ n\end{array}\right)$, such that $\omega_{j}^{N}=1$ is the differential operator of order zero in the vector $\Omega^{N}$. Construct the matrix $M$ of size $\left(\begin{array}{c}n+N \\ n\end{array}\right) \times\left(\begin{array}{c}N+n \\ n\end{array}\right)$, which is the identity matrix except for the fact that the $j$ th column is replaced by the vector $\Omega^{N}$. Note that $\operatorname{det} M=1$.

Let $\tilde{R}$ be a maximal square minor of the matrix $R_{\mu}\left(L_{1}, \ldots, L_{n+1}\right)$. Note that the elements in the $j$ th column of the matrix $\tilde{R} \cdot M$ are all monic monomial differential operators composed with the operators $L_{i}-\mu_{i}$. Then, expanding down this column while taking determinants, one finds exactly something of the form (2) with the coefficients of $D_{i}$ coming from the other minor determinants of $\tilde{R}$.

On the other hand, it is an elementary fact of linear algebra that $\operatorname{det} \tilde{R}=\operatorname{det} \tilde{R} \cdot M$ and so (2) must actually be equal to the order zero operator which is the partial $\mu$-shifted differential resultant of the operators $L_{i}$.

Now, suppose that $\psi\left(x_{1}, \ldots, x_{n}\right)$ satisfies each of the eigenvalue equations

$$
L_{i} \psi\left(x_{1}, \ldots, x_{n}\right)=\mu_{i} \psi\left(x_{1}, \ldots, x_{n}\right), \quad 1 \leq i \leq n+1
$$

Applying any linear combination of the operators $L_{i}-\mu_{i}$ to this gives zero, but we have by Lemma 3.1 that multiplying by the partial resultant, a function, is equivalent to applying such a combination. Then, we have the following corollary (a well-known result appearing also in [5]).

Corollary 3.1. If the operators $L_{i}$ have a common eigenfunction with eigenvalues $\mu_{i}$ then each partial $\mu$-shifted resultant vanishes.

\subsection{The commutative case}

As in the one-dimensional case, we will here show that the partial resultants provide polynomial equations satisfied by the operators $L_{i}$ in the case that they mutually commute. The remainder of the section will then be comprised of examples and counter examples of what we would hope to have as a consequence.

Now suppose that the operators $L_{i}(1 \leq i \leq n+1)$ mutually commute. By definition, any partial $\mu$-shifted differential resultant of these operators is a polynomial in the variables $\mu_{i}$ with coefficients possibly depending on the variables $x_{j}$. As a consequence of Lemma 3.1 we then find that the operators $L_{i}$ satisfy this polynomial.

Theorem 3.1. Let $p\left(\mu_{1}, \ldots, \mu_{n+1}\right)$ be any $\mu$-shifted differential resultant of the mutually commuting operators $L_{i}$, then

$$
p\left(L_{1}, \ldots, L_{n+1}\right)=0
$$

Proof. Only commutativity of the $\mu_{i}$ 's with the $L_{j}$ 's is required to rewrite $p\left(\mu_{1}, \ldots, \mu_{n+1}\right)$ in the form (2). So, since $\left[L_{i}, L_{j}\right]=0$ we can write $p\left(L_{1}, \ldots, L_{n+1}\right)$ by substituting $L_{i}$ for $\mu_{i}$ in (2). This, however, is clearly zero since every term has a factor of $L_{i}-\mu_{i}$ for some $i$. 
In the one-dimensional case, we can moreover say that the $\mu$-shifted differential resultant is a polynomial in $\mu_{1}$ and $\mu_{2}$ with constant coefficients or a multiple of such a polynomial by a function of $x_{1}$. Here, the results proved thus far leave open the possibility that the resultants will only produce polynomial equations satisfied by the operators with coefficients depending on the variables $x_{j}$. We were not able to produce any such examples or exclude the possibility.

\subsection{The zero possibility}

As stated in the introduction, given two commuting ODOs $L_{1}$ and $L_{2}$ the determinant of $R_{\mu}\left(L_{1}, L_{2}\right)$ (which happens to always be square in the case $n=1$ ) is a non-zero polynomial in $\mu_{1}$ and $\mu_{2}$ which is satisfied by the operators. Here we will see that the differential resultant does not always give such useful information in the higher dimensional case. Consider the case $n=2$ and

$$
L_{1}=\partial_{1}^{2}-\partial_{2}^{2}-1, \quad L_{2}=\partial_{1} \circ L_{1}, \quad L_{3}=\partial_{2} \circ L_{1} .
$$

Since these operators have constant coefficients, we can use Macaulay's definition of resultant as the greatest common divisor of all the partial resultants, and call that the differential resultant. Note that the operators satisfy the equation $L_{2}^{2}-L_{3}^{2}-L_{1}-L_{1}^{6}=0$ and so one might hope, given Theorem 3.1, that the differential resultant of these operators is $\mu_{2}^{2}-\mu_{3}^{2}-\mu_{1}-\mu_{1}^{6}$ (or at least is a non-zero multiple of this).

Lemma 3.2. The differential resultant of the operators $L_{i}$ is the zero polynomial in the variables $\mu_{i}(1 \leq i \leq 3)$.

Proof. One could, of course, merely compute the resultant according to the definition. However, there is a more direct and informative way to observe this fact. Since these operators are constant coefficient, the problem reduces to a problem of polynomial resultants. In particular, the resultant is the same as the resultant of the homogeneous polynomials

$$
\begin{aligned}
& p_{1}\left(x_{1}, x_{2}, x_{3}\right)=x_{1}^{2}-x_{2}^{2}-\left(\mu_{1}+1\right) x_{3}^{2}, \quad p_{2}\left(x_{1}, x_{2}, x_{3}\right)=x_{1}^{3}-x_{1} x_{2}^{2}-x_{1} x_{3}^{2}-\mu_{2} x_{3}^{3}, \\
& p_{3}\left(x_{1}, x_{2}, x_{3}\right)=x_{1}^{2} x_{2}-x_{2}^{3}-x_{2} x_{3}^{2}-\mu_{3} x_{3}^{3} .
\end{aligned}
$$

It is well known [15] that this resultant will be zero if these polynomials have a common zero in projective space. Although it is true that no "finite" point (with $x_{3} \neq 0$ ) is a common solution to these polynomials for all values of $\mu_{i}$, there are solutions at infinity. In particular, note that the point $(1,-1,0)$ satisfies all three polynomials regardless of the values of $\mu_{i}$.

It is interesting to note the geometry behind this situation. This problem of having a zero (differential) resultant never arises in the one-dimensional case essentially because only one point is being added at infinity in the $\left(x_{1}: x_{2}\right)$ line and that point is never a solution of the homogeneous polynomial. Whereas, in higher dimension, there is "room" at infinity for many solutions. Note that infinity, or $x_{3}=0$, is precisely where the condition becomes independent of the parameters $\mu_{i}$.

The same problem can also occur in a non-constant case, and so not simply an example of a polynomial resultant. In particular, the resultants of any three operators from the ring $R(K)$ described in Section 2.3.2 will be zero regardless of the values of the variables $\mu_{i}$. The mundane explanation of this fact here is merely that the $N$ th powers of $\partial_{1}$ and $\partial_{2}$ never appear in $\omega_{j}^{N-l_{i}} \circ\left(L_{i}-\mu_{i}\right)$ and so there are columns of the resultant matrix with all zero entries.

Recently, there have been significant advances in the theory of sparse resultants for polynomials which address these problems in the polynomial case (cf. [7], Chapter 7). If differential resultants prove to be useful in the case 
of commuting differential operators (as the next example indicates), then there is good reason for investigating a theory of sparse resultants in the differential case.

\subsection{Positive results}

A more encouraging example is given by the operators

$$
L_{1}=\partial_{1}^{2}-\partial_{2}^{2}, \quad L_{2}=x_{2} \partial_{1}+x_{1} \partial_{2}, \quad L_{3}=L_{1} \circ L_{2}-\gamma L_{1}, \quad \gamma \in \mathbb{C} .
$$

Given that $\left[L_{1}, L_{2}\right]=0$, as is easily checked, it is clear that $L_{3}$ also commutes with $L_{1}$ and $L_{2}$ and that the three together satisfy a polynomial equation $p\left(L_{1}, L_{2}, L_{3}\right)=0$ with

$$
p\left(\mu_{1}, \mu_{2}, \mu_{3}\right)=\mu_{3}-\mu_{1} \mu_{2}+\gamma \mu_{1} .
$$

Here, we compute a particular quotient of minors $D / A$, where $D$ is a partial resultant and $A$ turns out to depend on the $x$ variables alone. $D$ and $A$ are the minor determinants specified in [15], where it is proved that $D / A$ computes the greatest common divisor of the maximal minors. In our case, $D / A=p^{3}\left(\mu_{1}, \mu_{2}, \mu_{3}\right)$. This is very nearly what we would want, although there is presently no theory to explain the exponent " 3 " which arises.

It is intriguing that the result is independent of the variables $x_{1}$ and $x_{2}$ in this case. In the one-dimensional case, the resultant of two monic differential operators is independent of $x$ if and only if the operators commute. Here, the situation involves one operator, $L_{1}$, that has constant coefficients and others that do not, which cannot happen in the one-dimensional case.

\section{Acknowledgements}

Emma Previato gratefully acknowledges partial support under NSF Grant DMS-9971966.

\section{References}

[1] Yu. Berest, A. Kasman, D-modules and Darboux transformations, Lett. Math. Phys. 43 (1998) 279-294.

[2] A. Braverman, P. Etingof, D. Gaitsgory, Quantum integrable systems and differential Galois theory, Transform. Groups 2 (1997) 31-56.

[3] J.L. Burchnall, T.W. Chaundy, Commutative ordinary differential operators, Proc. Lond. Math. Soc. 211 (1923) 420-440.

[4] J.L. Burchnall, T.W. Chaundy, Commutative ordinary differential operators, Proc. R. Soc. A 118 (1928) 557-583.

[5] G. Carrà Ferro, A resultant theory for systems of linear partial differential equations, Lie Groups Appl. 1 (1) (1994) 47-55.

[6] O.A. Chalykh, A.P. Veselov, Commutative rings of partial differential operators and Lie algebras, Commun. Math. Phys. 125 (1990) 597-611.

[7] D. Cox, J. Little, D. O'Shea, Using Algebraic Geometry, Springer, Berlin, 1998.

[8] G. Darboux, Sur une proposition relative aux equation lineaires, Compt. Rend. 94 (1882) 1456.

[9] J.J. Duistermaat, F.A. Grünbaum, Differential equations in the spectral parameter, Commun. Math. Phys. 103 (1986) 177-240.

[10] F.A. Grünbaum, Bispectral Musings CRM Proceedings and Lecture Notes, Vol. 14, American Mathematical Society, Providence, RI, 1988, pp. 31-46.

[11] L. Hörmander, The Weyl calculus of pseudodifferential operators, Commun. Pure Appl. Math. 32 (1979) 360-444.

[12] E. Horozov, A. Kasman, Darboux transformations for bispectral quantum integrable systems, Lett. Math. Phys. 49 (1999) $131-143$.

[13] A. Kasman, Kernel inspired factorizations of partial differential operators, J. Math. Anal. Appl. 234 (2) (1999) 580-591.

[14] I.M. Krichever, The integration of nonlinear equations by methods of algebraic geometry, Funct. Anal. Appl. 12 (1977) 12-26.

[15] F.S. Macaulay, The algebraic theory of modular systems, Cambridge Tracts Math. Math. Phys. 19 (1916) 13.

[16] V.B. Matveev, M.A. Salle, Darboux transformations and solitons, Springer Series on Nonlinear Dynamics, Springer, Berlin, 1991.

[17] A. Nakayashiki, Structure of Baker-Akhiezer modules of principally polarized abelian varieties, commuting partial differential operators and associated integrable systems, Duke Math. J. 62 (2) (1991) 315-358.

[18] A. Nakayashiki, Commuting partial differential operators and vector bundles over abelian varieties, Am. J. Math. 116 (1) (1994) 65-100. 
[19] E. Previato, Another algebraic proof of Weil's reciprocity, Atti Accad. Naz. Lincei Cl. Sci. Fis. Mat. Natur. Rend. (9) Mat. Appl. 2 (2) (1991) 167-171.

[20] E. Previato, Seventy years of spectral curves: 1923-1993, Integrable Systems and Quantum Groups (Montecatini Terme, 1993), Lecture Notes in Mathematics, Vol. 1620, Springer, Berlin, 1996, pp. 419-481.

[21] F.J. Plaza Martin, Generalized KP Hierarchy for Several Variables, Preprint math/0008004.

[22] M.A. Shubin, in: M. Hazewinkel (Ed.), Pseudo-differential Operator, Entry in Encyclopaedia of Mathematics: An Updated and Annotated Translation of the Soviet Mathematical Encyclopaedia, Kluwer Academic Publishers, Dordrecht, MA, 1988. 